\title{
Comportamiento agronómico del plátano (Musa paradisiaca L.) cv. CEMSA $3 / 4$ mediante la selección de cormos en base a productividad en Potosí, Rivas 2015
}

\section{Agronomic performance of banana plantain (Musa paradisiaca L.) cv. CEMSA $3 / 4$ by selecting corms based on productivity Potosí, Rivas, 2015}

\author{
Ronald Rivera Rivas, Samuel Vaca Suárez¹, Víctor Aguilar Bustamante² \\ ${ }^{1}$ Ingenieros agrónomos \\ ${ }^{2}$ Doctor en Ciencias, profesor titular de la Universidad Nacional Agraria, ORCID: https://orcid.org/0000-0002-8356-739X
}

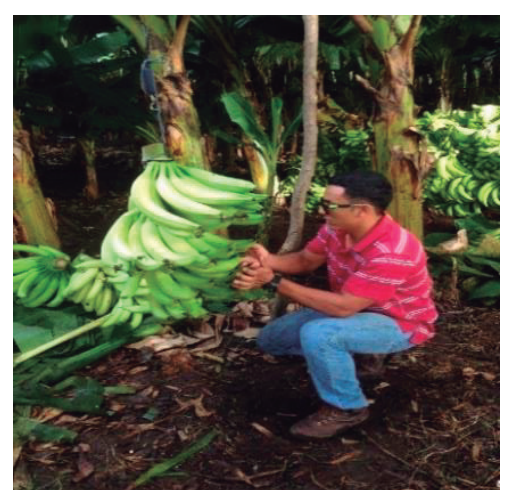

RESUMEN

Se evaluó el comportamiento agronómico del plátano (Musa paradisiaca L.) cv. CEMSA $3 / 4$ en plantas con cormos seleccionados de plantas con racimos igual o mayor a 40 dedos (Superior o tratamiento 1) y plantas seleccionadas al azar por el productor (Testigo o tratamiento 2). El estudio se realizó en la finca "San Antonio" Potosí, Rivas. Cada tratamiento consistió de 10 surcos con 25 plantas a una distancia de $2.4 \mathrm{~m}$ entre surcos y $1.3 \mathrm{~m}$ entre plantas para una densidad poblacional de 3200 plantas por hectárea. Las parcelas fueron establecidas el 23 de octubre del 2014 y la cosecha se terminó en diciembre 2015. Se realizó la prueba de T student para dos poblaciones independientes con 5\% de margen de error y correlación múltiple. El programa utilizado fue MINITAB 17. El peso de racimo fue igual $(\mathrm{P}=0.714)$ para ambas parcelas con $20.44 \mathrm{~kg}$ para la parcela testigo y $20.31 \mathrm{~kg}$ para la parcela superior. El número de manos fue de 10.06 para la parcela testigo y 10.39 para la superior $(\mathrm{P}=0.002)$. El número de dedos fue de 51.51 para la testigo y 52.95 para la superior $(\mathrm{P}=0.019)$ superando en un dedo por racimo la parcela superior. La longitud del dedo central de la segunda mano fue $32.45 \mathrm{~cm}$ para la testigo y para la superior de $31.74 \mathrm{~cm}(\mathrm{P}=0.016)$. El grosor del dedo $(\mathrm{P}=0.006)$ fue de $15.67 \mathrm{~cm}$ para la testigo y $15.35 \mathrm{~cm}$ para la superior. El peso promedio de cada dedo fue para la parcela testigo de $367.0 \mathrm{~g}$ y para la parcela superior de $344.9 \mathrm{~g}$. Se obtuvieron rendimientos de $58.86 \mathrm{tha}^{-1}$ en la parcela testigo y $58.49 \mathrm{t} \mathrm{ha}^{-1}$ en la parcela superior. El ensayo continuará con la cosecha de 2016 y 2017. Palabras claves: cormo, plátano, racimo, manos, dedos y rendimiento.

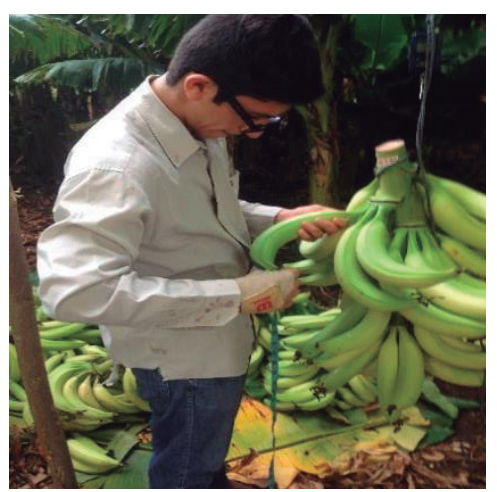

\section{ABSTRACT}

A field experiment was carried out at San Antonio Farm located in Potosí, Rivas from October 2014 to December 2015. Two experimental plots were established with 250 plants each. The variety of banana plantain (Musa paradisiaca L.) cv. CEMSA 3/4 was used. The experiment was established on October $23^{\text {th }}, 2014$. Suckers from plants with bunch with 40 or more fingers was selected to establish the superior plot (Treatment 1). In the test plot the suckers was selected by the farmer (Treatment 2). Rows with 25 plants at 2.4 $\mathrm{m}$ between rows and $1.3 \mathrm{~m}$ among plants for a density of 3200 plants per hectare. T-test per independent plots and multiple correlation were used to analyze the data. The bunch weigh was $20.44 \mathrm{~kg}$ for test plot and $20.31 \mathrm{~kg}$ for superior plot $(\mathrm{P}=0.714)$. The number of hand per bunch were 10.06 per test plot and 10.39 for the superior $(\mathrm{P}=0.002)$. The number of fingers per bunch was 51.51 per test plot and 52.95 for the superior $(\mathrm{P}=0.019)$. The finger length was 32.45 $\mathrm{cm}$ for the test plot and $31.74 \mathrm{~cm}$ for the superior plot $(\mathrm{P}=0.016)$. The diameter of finger was $15.67 \mathrm{~cm}$ for test plot and $15.35 \mathrm{~cm}$ for the superior plot $(\mathrm{P}=0.006)$. The mean weigh of finger was 367.0 $\mathrm{g}$ test plot and $344.9 \mathrm{~g}$ for superior plot. The total yield was 58.86 $\mathrm{t} \mathrm{ha}^{-1}$ in the test plot and $58.49 \mathrm{t} \mathrm{ha}^{-1}$ in the superior plot. The field experiment will continue with the harvest 2016 and 2017.

Keywords: Sucker, plantain, bunch, hands, fingers and yield.
Recibido: 26 de enero del 2018 Aceptado: 24 de mayo del 2018

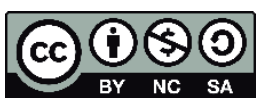

Los artículos de la revista La Calera de la Universidad Nacional Agraria, Nicaragua, se comparten bajo términos de la licencia Creative Commons: Reconocimiento, No Comercial, Compartir Igual. Las autorizaciones adicionales a las aquí delimitadas se pueden obtener en el correo freddy.aleman@ci.una.edu.ni

(C) Copyright 2018. Universidad Nacional Agraria 


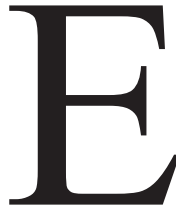

1 cultivo de plátano (Musa paradisiaca L.) es de los rubros más importantes en Nicaragua puesto que juega un papel fundamental en la dieta alimenticia y en la economía nacional, generando alrededor de 130,000 empleos y divisas por las exportaciones realizadas a Centroamérica, Puerto Rico y Estados Unidos (Quintero, 2013). Según cifras del Centro de Trámite de las Exportaciones (CETREX, 2014), para enero-diciembre 2014 la exportación de plátanos y bananos generaron al país alrededor de 9.2 millones de dólares en ingresos. El plátano se produce en todo el territorio nacional destacando Rivas como el mayor productor de plátanos con casi 375 millones de unidades con un área de siembra de 6,300 ha y con un rendimiento promedio de 59,143 unidades por hectárea (Quintero, 2013).

La selección de material de siembra en base a características de productividad o sea mayor número de dedos y peso por dedo, poca disponibilidad de agua para riego y el manejo inadecuado de las plantaciones son las principales limitantes que tienen los productores (Galo, 2013). Es por ello que productores de Rivas y León en conjunto y con el apoyo de la Universidad Nacional Agraria (UNA) y Universidad Nacional Autónoma de Nicaragua sede León (UNAN-León) han iniciado trabajos de investigación donde se implementan técnicas de marcado de los hijos de plátano de plantas madres con racimo con 40 o más dedos de excelente calidad.

Trabajos similares se están realizando en República Dominicana, Panamá y Ghana en África para desarrollar un programa de mejoramiento genético con fondos provenientes de Bioversity International (BI-CGIAR). En Potosí, Rivas se establecieron estas dos parcelas de plátano variedad CEMSA $3 / 4$ seleccionando hijos de plantas madres a partir de 40 o más dedos por racimo (parcela superior) y compararlas con la selección tradicional que hacen los plataneros de Rivas que seleccionan los mejores hijos de sus plantaciones sin tomar en cuenta el peso del racimo y el número de dedos (Parcela testigo).

\section{MATERIALES Y MÉTODOS}

Ubicación del estudio. El estudio se realizó en la finca "San Antonio" ubicada en el municipio de Potosí, departamento de Rivas, propiedad de don Genaro Martínez, productor afiliado a la Asociación de Plataneros de Rivas (APLARI). La finca se encuentra ubicada en la parte sur del litoral Pacífico de Nicaragua, con una altitud de 57 msnm entre las coordenadas $11^{\circ} 26^{\prime}$ latitud norte y $85^{\circ} 49^{\prime}$ longitud oeste. Temperatura promedio de $27^{\circ} \mathrm{C}$, precipitaciones anuales de $1400 \mathrm{a} 1500 \mathrm{~mm}$ año-1 y humedad rela- tiva en el ambiente que oscilan entre 40 a $75 \%$ en época seca y de 80 a $100 \%$ en el período de lluvias (Castellón, 2009). Posee suelo franco de origen volcánico (andisol) característico de la región, $\mathrm{pH}$ de 7.2 y $3.1 \%$ de MO.

Diseño metodológico. El ensayo se estableció en parcelas independientes, sin repeticiones. Cada tratamiento constó de 10 surcos y 25 plantas por surco. La siembra fue a 2.4 m entre surcos y 1.3 metros entre plantas para una densidad poblacional de 3200 plantas/ha. El tratamiento testigo el productor selección los hijos al azar a como lo hacen todos los productores y en el tratamiento 2 o parcela superior los hijos se seleccionaron cuando la planta madre tenía 40 más dedos. Los hijos se marcaron con una cinta al momento de la cosecha. Posteriormente al sembrar una nueva plantación se seleccionaban estos hijos marcados con la cinta plástica.

\section{Variables evaluadas}

Peso del racimo (kg). Una vez cosechado se pesó cada racimo (con raquis) con una balanza electrónica portátil marca CIL.

Número de manos por racimo. Se contaron el número de manos por racimo partiendo de arriba hacia abajo.

Número de dedos por racimo. Se contaron los dedos o frutos por racimo.

Longitud del fruto (cm). Una vez realizada la cosecha se determinó la longitud del fruto central de la segunda mano usando una cinta plástica.

Grosor del fruto (cm). La medición del diámetro se efectuó en el centro de la curva del fruto o dedo central de la segunda mano usando una cinta plástica.

Peso del fruto (g). El dedo central que se le midió el largo y el grosor también se pesó usando una balanza electrónica.

Rendimiento total (t ha $\left.\mathbf{~}^{-1}\right)$. Una vez cosechada la parcela se procedió a sacar promedios de peso del racimo de cada tratamiento, se extrapoló de acuerdo a las densidades de siembra por hectárea utilizadas en el área de estudio, asumiendo un $10 \%$ de pérdidas para luego convertirla a toneladas por hectárea.

Análisis estadístico. La base de datos se construyó en hojas electrónicas de Microsoft Excel 2010. La información 
se procesó a través del software SPSS v.22. Se realizó una prueba de $\mathrm{T}$ student para muestras de poblaciones independientes $(\alpha=0.05)$ para las variables de rendimiento.

\section{RESULTADOS Y DISCUSIÓN}

Peso de racimo (kg). Se registraron racimos de 20.31 para la parcela superior y $20.44 \mathrm{~kg}$ para la parcela testigo. (Tabla 1). No se encontró diferencia significativa en el rendimiento $(\mathrm{P}=0.714)$. Estos pesos están muy por encima de los reportados anteriormente por Martínez y González (2007). Ellos encontraron rendimientos inferiores a los encontrados en la parcela de Potosí, Rivas con un peso del racimo entre 7 y $13 \mathrm{~kg}$. En ensayos establecidos por la UNAN León han encontrado racimos con peso de $23.1 \mathrm{~kg}$ en León, $14 \mathrm{~kg}$ en Telica y $16.9 \mathrm{~kg}$ en posoltega.

El raquis es conocido como "pinzonte" y no tiene ningún valor comercial, sin embargo, en él se concentran grandes cantidades de nutrientes los cuales pueden ser reincorporados al suelo de las plantaciones. Los frutos se dividen en comerciales o de primera, colas o de segunda y pirachas o dedos de tercera (figura 1)

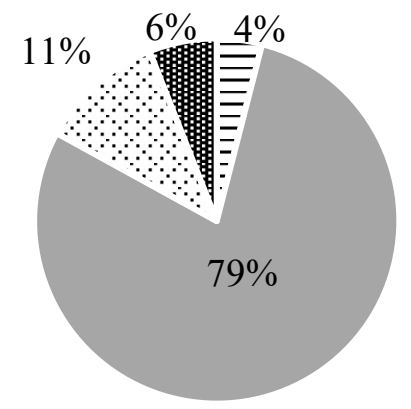

10.06 manos por racimo (Tabla 1). El rango del número de manos estuvo entre 8 y 12 manos.

Número de dedos por racimo. En cuanto al número de dedos por racimo, la parcela superior alcanzó 52.95 dedos y la parcela testigo 51.51 dedos por racimo $(\mathrm{P}=0.019)$. Estos valores están muy por encima de los registrados por Martínez y González (2007) que indican que el cv. CEMSA $3 / 4$ alcanza valores promedio de 46 dedos por racimo (Cuadro 1). En León se han encontrado 46 dedos por racimo, en Telica, León se han encontrado 41 dedos por racimo y en Posoltega, León 33 dedos por racimo estableciendo estos mismos ensayos sembrando hijos de plátano con 40 o más dedos por racimo.

Longitud de fruto (cm). Los dedos de la parcela superior alcanzaron una longitud de $31.74 \mathrm{~cm}$ y las de la parcela testigo $32.45 \mathrm{~cm}(\mathrm{P}=0.016)$ (cuadro 1$)$.

Grosor del fruto (cm). El grosor de los dedos de la parcela superior fue de $15.35 \mathrm{~cm}$ y los de la parcela testigo de 15.67 ( $\mathrm{P}=0.006)$ (cuadro 1).

Figura 1. Distribución de los tipos de dedos en el racimo o raquis en la parcela superior izquierda y testigo a la derecha.

Número de manos por racimo. Se encontraron diferencias significativas en el número de manos $(\mathrm{P}=0.002)$. La parcela superior tuvo 10.39 manos y la parcela testigo
Rendimiento total ( $\left.\mathbf{t} \mathbf{h a}^{-1}\right)$. La mayor parte de la producción mundial de plátano está destinada a suplir el consumo interno de los países productores y tan solo una pequeña

Cuadro 1. Peso promedio de racimo, Número promedio de manos por racimo, Número promedio de dedos por racimo, Longitud promedio de dedo central de la segunda mano, y Grosor promedio de dedo central de la segunda mano de plantas de plátano cv. CEMSA 3/4 bajo dos métodos de selección de cormos, Potosí, Rivas, 2015

\begin{tabular}{cccccc}
\hline $\begin{array}{c}\text { Promedio } \\
\text { central de mano }\end{array}$ & Peso racimo $(\mathrm{kg})$ & $\begin{array}{c}\text { Número de manos } \\
\text { por racimo }\end{array}$ & $\begin{array}{c}\text { Número de dedos } \\
\text { por racimo }\end{array}$ & $\begin{array}{c}\text { Longitud de dedo cen- } \\
\text { tral de la segunda mano }\end{array}$ & $\begin{array}{c}\text { Grosor de dedo de } \\
\text { la segunda mano }\end{array}$ \\
\hline Parcela testigo & $20.44 \mathrm{a}$ & $10.06 \mathrm{a}$ & $51.51 \mathrm{~b}$ & $32.45 \mathrm{a}$ & $15.67 \mathrm{a}$ \\
Parcela superior & $20.31 \mathrm{a}$ & $10.39 \mathrm{~b}$ & $52.95 \mathrm{a}$ & $31.74 \mathrm{~b}$ & $15.35 \mathrm{~b}$ \\
\hline
\end{tabular}


parte es comercializada en los mercados internacionales (MIFIC, 2007). Los rendimientos promedios para Nicaragua al año 2006 fueron de $9.78 \mathrm{t} \mathrm{ha}^{-1}$ considerados relativamente bajos.

Teniendo en cuenta el número de dedos promedios por racimo y la densidad de siembra de 3200 plantas $\mathrm{ha}^{-1} \mathrm{y}$ asumiendo un $10 \%$ de pérdidas de plantas que son suprimidas por distintos factores dentro de la población se pueden obtener un estimado de los rendimientos logrados en cada plantación por cada hectárea de cultivo. Para la parcela superior de 152496 dedos por hectárea y para la parcela testigo de 148348 dedos por hectárea habiendo una diferencia de 4148 dedos que a un precio promedio de 3 córdobas suman un total de 12444 córdobas.

Resultados similares en estas mismas variables fueron obtenidos por Salgado y Ruiz (2015) y también por Serrano y Valdivia (2015), quienes de igual forma evaluaron la selección de material de siembra a través de características productivas en el municipio de Telica, León, Nicaragua.

\section{CONCLUSIONES}

Se encontró que las plantas seleccionadas en base a productividad presentaron una altura de hijo más alto. En general se encontró que las plantas más precoces fueron más productivas y que las plantas seleccionadas al azar por el productor son más precoces. No se encontró diferencia significativa en el peso del racimo para ambas tecnologías. El número de dedos por racimo fue mayor en la parcela superior y la longitud y el grosor del dedo central de la segunda mano fue superior en la parcela testigo.

\section{RECOMENDACIONES}

Evaluar el segundo ciclo 2016 y el tercer ciclo 2017 del cultivo para conocer si el comportamiento productivo de la primera generación es heredado a la segunda y tercera cosecha. Establecer una nueva parcela con hijos de plantas madres con 50 o más dedos. Enseñar a los productores a marcar las plantas con alta productividad para posteriormente seleccionar sus hijos y uniformizar su plantación.

\section{REFERENCIAS BIBLIOGRÁFICAS}

Castellón, JD. 2009. Estudio de poblaciones de fitonematodos, nematodos de vida libre, hongos endofíticos y su relación con propiedades físicas y químicas del suelo en el cultivo del plátano en Rivas - Nicaragua. Tesis MSc. Agricultura Ecológica. Turrialba, CR. Centro Agronómico Tropical de Investigación y Enseñanza. 93 p.

CETREX. 2014. Exportaciones autorizadas periodo enero-diciembre 2013-2014 (en línea). Consultado 18 oct 2015 . Disponible en: http://www.cetrex.gob.ni/website/servicios/tproduc14.html

Galo Romero, H. 2013. Musáceas: otra mina por explotar. (En línea). La Prensa. Consultado 10 nov. 2014, disponible en http://www. laprensa.com.ni/2013/11/27/economia/171894-musaceas-otra-mina-por-explotar

Martínez, E; González, M. 2007. Instructivo técnico del cultivo del plátano. CU.P. 8

Ministerio de Fom ento, Industria y Comercio (MIFIC). 2007. Ficha del Plátano. Managua, NI. 21 p.

Quintero, L. 2013. Rivas principal exportador de plátano (en línea). El Nuevo Diario. Consultado 4 nov. 2014, disponible en http:// www.elnuevodiario.com.ni/economia/275024

Salgado, T; Ruiz, M. 2015. Evaluación del desarrollo fenológico y productividad de plantas elites de plátanos Hartón enano (AAB) multiplicados en cámaras térmicas y establecidas en La finca El Pegón UNAN-León 2014-2015. Tesis Ing. Agroecología Tropical. León, NI. Universidad Nacional Autónoma de Nicaragua-León. 47 p.

Serrano, V; Valdivia, A. 2015. Evaluación fenológica y productiva de plantas superiores en el cultivo de plátano Hartón enano (AAB) en el municipio de Telica, 2 ciclo periodo 2014-2015. Tesis Ing. Agroecología Tropical. León, NI. Universidad Nacional Autónoma de Nicaragua-León. 62 p. 